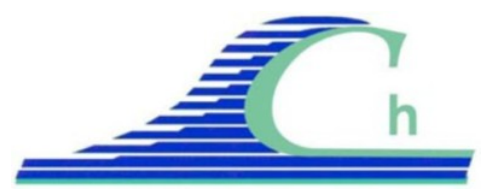

XII ${ }^{\text {ìmes }}$ Journées Nationales Génie Côtier - Génie Civil

Cherbourg, 12-14 juin 2012

DOI:10.5150/jngcgc.2012.024-C C Editions Paralia CFL

disponible en ligne - http://www.paralia.fr - available online

\title{
Morphodynamique des barres sableuses de déferlement le long des plages en baie
}

\author{
Bruno CASTELLE ${ }^{1}$, Giovanni COCO $^{2}$
}

1 Université de Bordeaux, UMR 5805 CNRS - EPOC,

Avenue des Facultés, 33405 Talence Cedex, France.

b.castelle@epoc.u-bordeaux1.fr

2 Environmental Hydraulics Institute "IH Cantabria", Universidad de Cantabria, c /Isabel Torres n 15.39011, Santander, Espagne.

giovanni.coco@unican.es

\section{Résumé :}

Dans cette étude nous utilisons un modèle morphodynamique non-linéaire qui permet de reproduire les circulations en zone de déferlement et, pour la première fois, les évolutions morphologiques couramment observées le long des plages en baie. Nos simulations suggèrent que l'influence des caps sur la morphodynamique des barres peut se propager progressivement dans l'ensemble du domaine. Une obliquité instationnaire du champ de vague induit des appariements et scissions permanentes des systèmes barre/chenal et une grande variabilité des longueurs d'onde le long de la plage. Cette variabilité le long de la plage est consistante avec l'hypothèse que les systèmes barre/chenal le long des plages en baie sont des motifs auto-organisés et est en accord avec de récentes observations de la dynamique des systèmes barre/chenal dans les plages en baie.

\section{Mots-clés :}

Morphodynamique - Auto-organisation - Dynamique non-linéaire - Chenal de cap

\begin{abstract}
We use a nonlinear morphodynamic model that can reproduce the flow circulation and, for the first time, the morphological characteristics observed on natural embayed beaches. Our simulations also suggest that the influence of the headlands can progressively propagate into the whole domain. A time-varying wave angle also results in persistent attraction of rip channels with headland rips, the splitting of shoals, an increase in merging of rip channels and more alongshore-variable rip spacing. The longshore variability of rip channel wavelength along embayed beaches is consistent with the hypothesis that rips are self-organized patterns and is in line with recent field observations.
\end{abstract}

\section{Keywords:}

Morphodynamics - Self-organization - Nonlinear dynamics - Headland rip 


\section{Introduction}

Les plages sableuses en baie sont des plages qui peuvent être de dimensions variables, généralement délimitées par des avancées rocheuses (ou plus rarement par des ouvrages de protection côtière). Généralement, il y a peu ou pas d'échanges hydro-sédimentaires entre les plages en baie et les littoraux adjacents, mis à part pendant les épisodes de tempête. Ainsi, la dynamique des plages en baie peut parfois être appréhendée à la manière d'un système fermé. La taille et la dynamique des barres sableuses de déferlement tridimensionnelles (systèmes de type barre/chenal ou en croissant) est souvent contrainte par les dimensions longitudinales de la plage. Alors que ces barres sableuses sont un élément essentiel du système plage, le rôle des contraintes longitudinales des plages en baie sur la dynamique des barres de déferlement tridimensionnelles est encore très mal compris.

SHORT \& MASSELINK (1999) ont synthétisé les circulations/morphologies typiquement observées le long des plages en baie : (1) 'circulation normale' caractérisée par des systèmes barre/chenal ayant un comportement similaire à celui observé sur les plages ouvertes (e.g., GALLOP et al., 2011), avec la présence de chenaux contre chaque cap (headland rip) ; (2) 'circulation cellulaire' où l'on observe un chenal contre chaque cap rocheux (e.g., LOUREIRO et al., sous presse), ou un chenal dans le centre de la plage ; (3) 'circulation transitoire', où la topographie et l'hydrodynamique influence le comportement des systèmes barre/chenal (e.g., ENJALBERT et al., 2011).

Alors que les études numériques sur la formation et la dynamique des systèmes barre/chenal sont nombreuses le long des plages ouvertes (e.g., GARNIER et al., 2008 ; CASTELLE \& RUESSINK, 2011), aucune modélisation morphodynamique n’a été entreprise afin d'expliquer les mécanismes qui contrôlent la formation et l'évolution des systèmes de barres le long des plages en baie. Une exception notable est l'étude de RENIERS et al. (2004) qui n'ont toutefois pas étudié de manière systématique l'impact des caps sur la morphodynamique des barres. Pourtant, alors que l'on sait désormais que sur les plages ouvertes les ondes de bord stationnaires ne sont pas à l'origine de la présence des systèmes barre/chenal ou en croissant, mais que leur dynamique est contrôlée par des mécanismes d'auto-organisation (COCO \& MURRAY, 2007), le doute persiste pour les plages en baie. En effet, la présence des ondes de bord stationnaires est très largement favorisée par les caps rocheux aux deux extrémités de la plage. Dans ce papier nous utilisons un modèle morphodynamique non-linéaire pour examiner pour la première fois la formation et la dynamique non-linéaire des systèmes barre/chenal le long des plages sableuses en baie.

\section{Modèle morphodynamique}

Le modèle morphodynamique non-linéaire utilisé ici (CASTELLE \& RUESSINK, 2011) couple le modèle spectral SWAN (BOOIJ et al., 1999) avec un modèle de circulation classiquement forcé par les tensions de radiation, et un modèle de transport 


\section{XII ${ }^{\text {èmes }}$ Journées Nationales Génie Côtier - Génie Civil \\ Cherbourg, 12-14 juin 2012}

sédimentaire. Dans ce dernier, nous utilisons l'approche d' «état de base » (e.g., GARNIER et al., 2008), qui fait l'hypothèse que les flux sédimentaires (vers la plage) induits par les non-linéarités des vagues et ceux (vers le large) induits par le courant de retour sont en équilibre pour un profil de plage donné (« état de base »). En essence, l'approche considère que les temps d'évolution caractéristiques des chenaux et barres en croissant sont plus courts que les échelles de temps associées aux évolutions du profil de plage moyen dans l'axe transversal. Pour plus d'information sur cette approche, le lecteur est invité à consulter, par exemple, GARNIER et al. (2008). A travers une gestion adéquate du trait de côte, il est à noter que le modèle utilisé ici est à l'heure actuelle le seul qui permette de suivre la formation, l'évolution non-linéaire et la saturation des barres sableuses tridimensionnelle de type barre/chenal et barre en croissant, ainsi que les oscillations du trait de côte associées (CASTELLE \& RUESSINK, 2011).

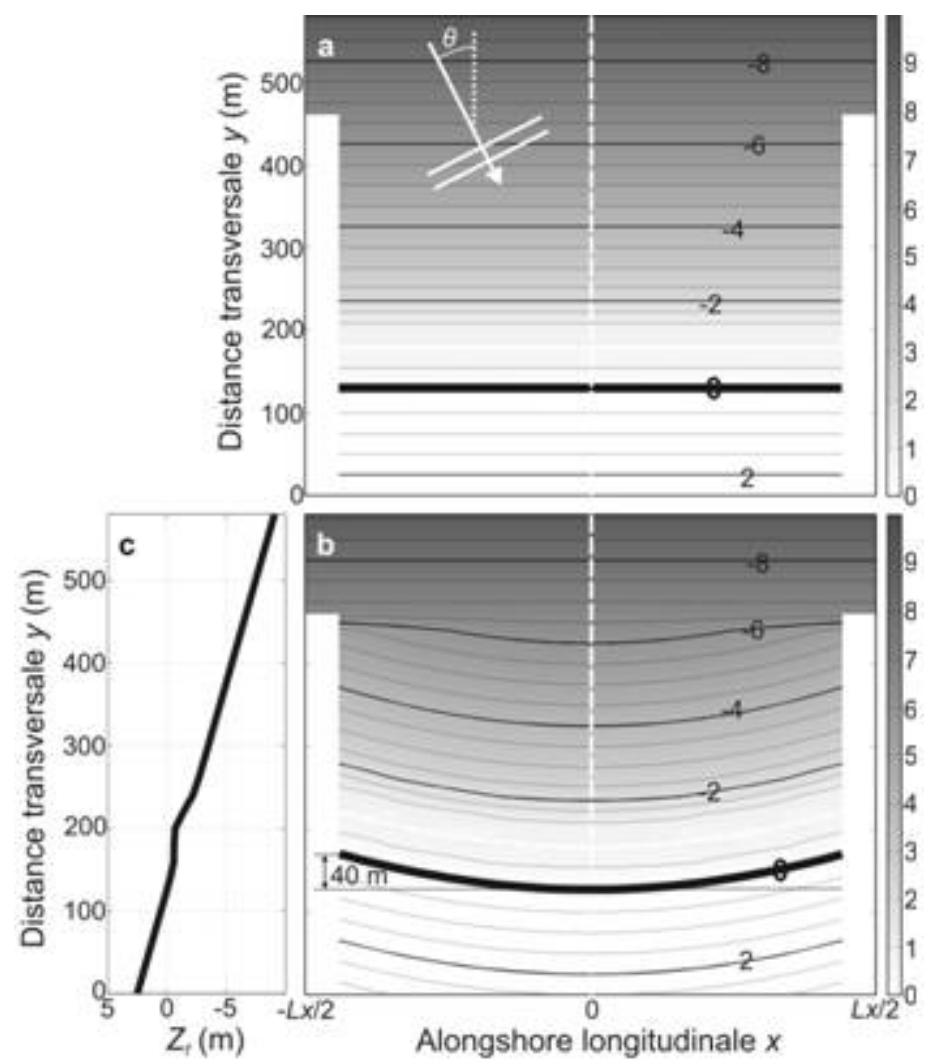

Figure 1.Bathymétries initiales (i.e. état de base) de plages en baie. (a) Plage rectiligne, (b) plage incurvée et (c) profil transversal.

Nous utilisons ce modèle en partant d'un grand nombre de bathymétries idéalisées de plages en baie contraintes par 2 caps rocheux, caractérisées par une longueur $L x=500$, 1000, 2000, 4000 et $8000 \mathrm{~m}$ et différents degrés de courbure (figure 1). Des forçages de vagues stationnaires avec une hauteur significative $H_{s}=1 \mathrm{~m}$, une période pic $T p=10 \mathrm{~s}$ et 
un angle d'incidence $\theta=0^{\circ}, 5^{\circ}$ et $10^{\circ}$, ainsi que des forçages de vagues instationnaires caractérisés par des variations sinusoidales de $\theta$ sur une période de 4 jours et d'amplitude $5^{\circ}$ ou $10^{\circ}$, sont considérés. Les simulations sont réalisées sur des périodes de 60 jours. Afin de s'affranchir des problèmes liés à la rotation des plages en baie exposées aux houles obliques (e.g., HARLEY et al., 2011), les 20 premiers jours de simulation sont étudiés en détails ici. Afin d'étudier l'évolution spatio-temporelle des chenaux, on utilise l'évolution temporelle du profil longitudinal de plage $Z b(x, t)=Z f(x, y=180 \mathrm{~m}, t)$ avec $Z f$ l'élévation du fond sableux (figure 1 ).

\section{Résultats}

\subsection{Climats de houle stationnaires}

La figure 2 montre un exemple de formation et d'évolution de systèmes barre/chenal pour des vagues stationnaires avec $H s=1 \mathrm{~m}, T p=10 \mathrm{~s}$ en $\theta=5^{\circ}$ en partant d'une plage incurvée avec $L x=2000 \mathrm{~m}$. Les résultats montrent la formation de chenaux de cap à chaque extrémité de la baie, avec également la formation de chenaux au centre de la baie qui s’apparient tout en migrant dans le sens de la dérive littorale.
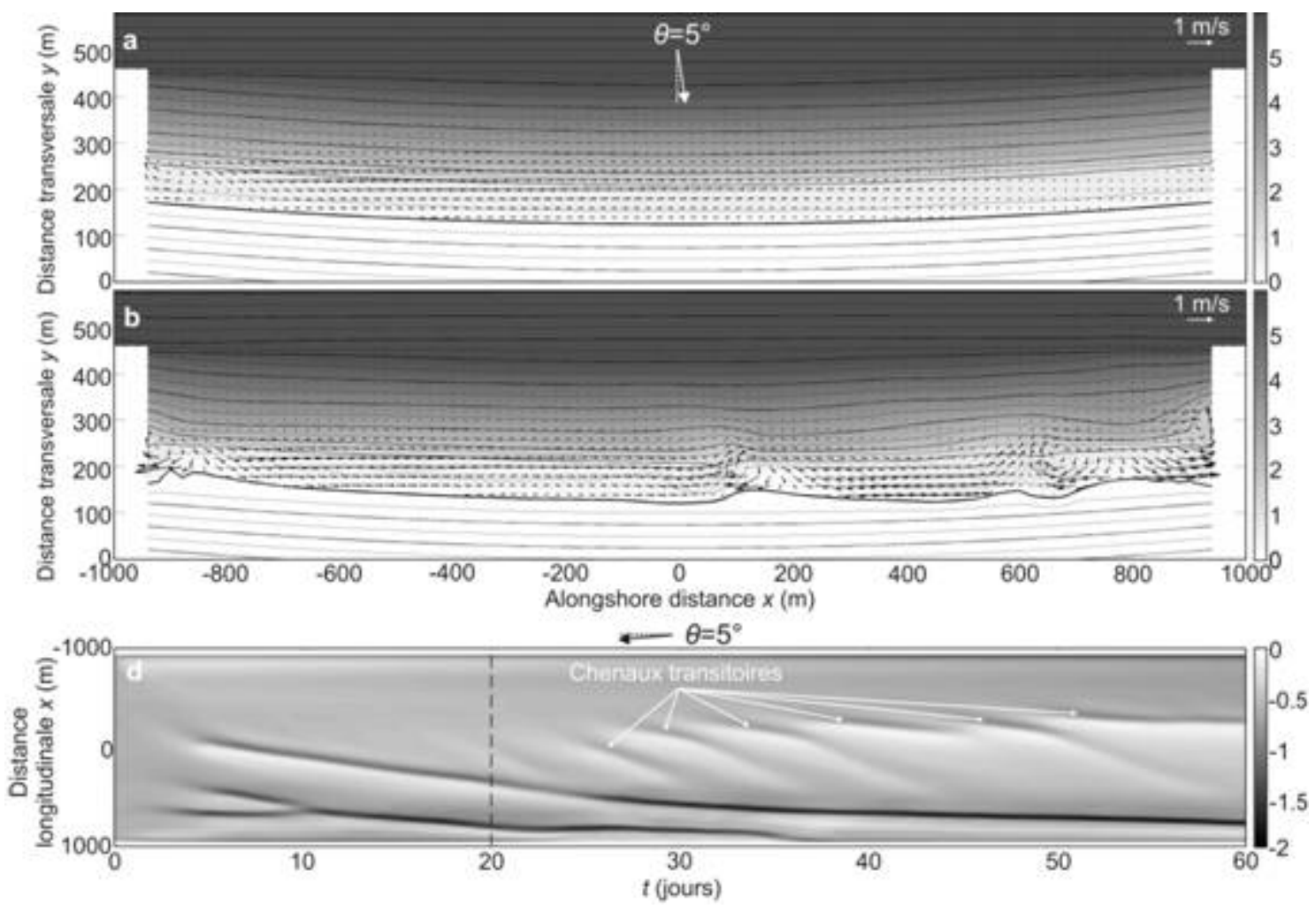

Figure 2. Evolution d'une plage en baie incurvée $(L x=2000 \mathrm{~m})$ soumise à des vagues avec $H s=1 \mathrm{~m}, T p=10$ s et $\theta=5^{\circ}$ avec la morphologie et les courants à (a) $t=0$, (b) $t=10$ jours et (c) évolution temporelle du profil longitudinal $\mathrm{Zb}$. 

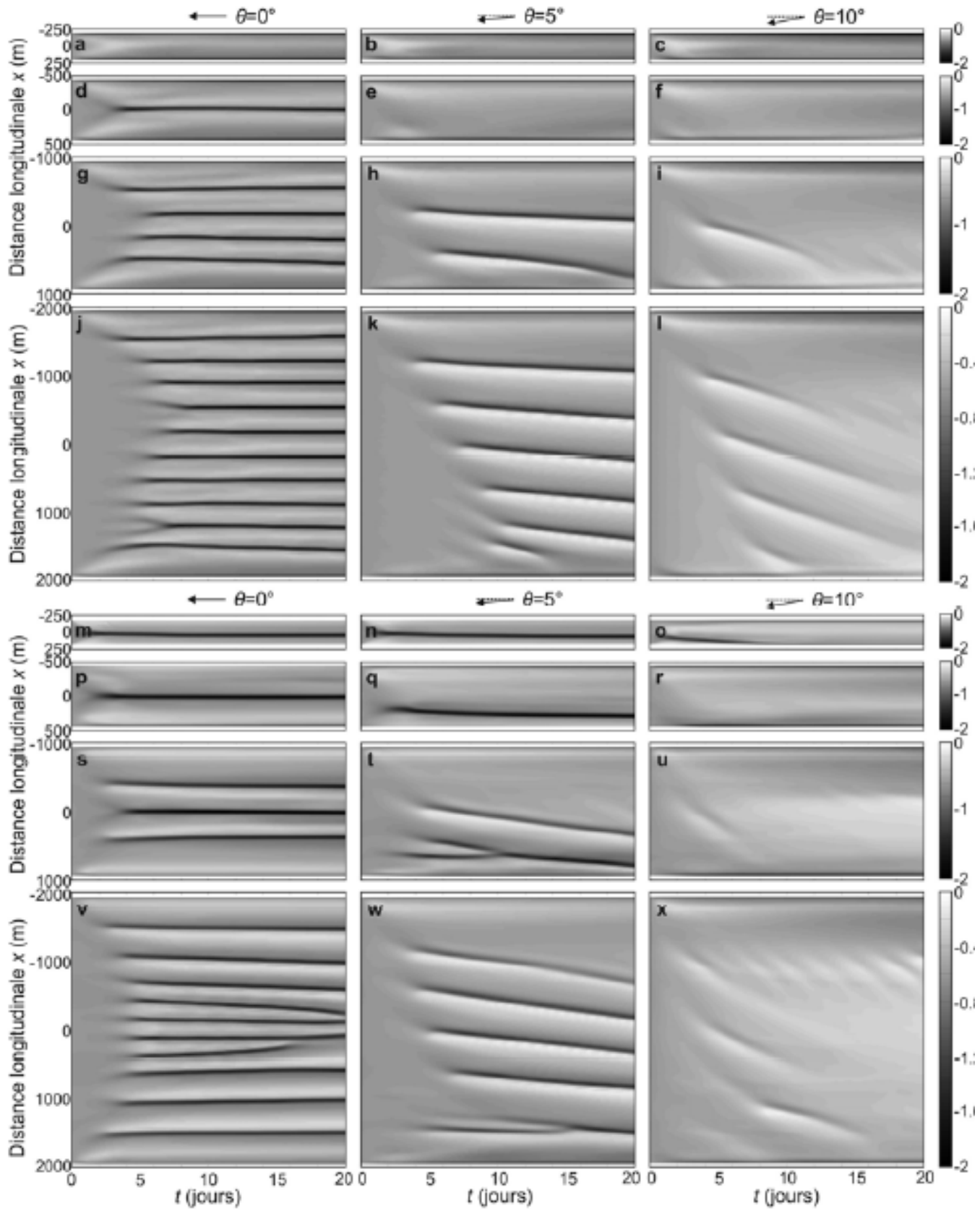

Figure 3. Evolution temporelle du profil longitudinal $\mathrm{Zb}$ pour des forçages de vagues stationnaires et des plages en baie (a-l) linéaires et (m-x) incurvées.

Le figure 3 synthétise les évolutions de profil longitudinal $Z b$ pour l'ensemble des forçages stationnaires (les simulations pour $L x=8000 \mathrm{~m}$ ne sont pas montrées ici). Les chenaux de cap sont systématiquement observés pour les plages initialement linéaires et sont absents pour les plages à forte courbure (e.g., figure $3 \mathrm{~m}, \mathrm{n}, \mathrm{q}, \mathrm{p}$ ). Pour une condition de vagues donnée, la longueur d'onde des chenaux est systématiquement la même ou plus grande pour les plages incurvées que pour les plages linéaires. 


\subsection{Climats de houle instationnaire}

La figure 4 montre quelques évolutions de $Z b$ pour des forçages de vagues instationnaire en angle d'incidence $\theta$ (amplitude de $10^{\circ}$ ) pour des plages initialement linéaires avec $L x=500,1000,2000$ et $4000 \mathrm{~m}$. Lorsque les dimensions de la plage en baie sont suffisamment larges pour développer plusieurs chenaux de vidange (i.e., figures $4 c$ et d), on remarque que les chenaux proches des caps sont "aspirés" par le chenal de cap. Cela, en cascade, implique des longueurs d'onde de système plus grandes au centre de la baie. Puisque, intrinsèquement, les systèmes auto-organisés tendent systématiquement à s'auto-organiser dans une longueur d'onde régulière et correspondante à un forçage de vagues donné, cela implique des scissions de barres. Ainsi, la dynamique non-linéaire (scissions et appariements) des systèmes barre/chenal est exacerbée par la présence des caps, ce qui implique également de fortes variabilités spatio-temporelles des longueurs d'onde.

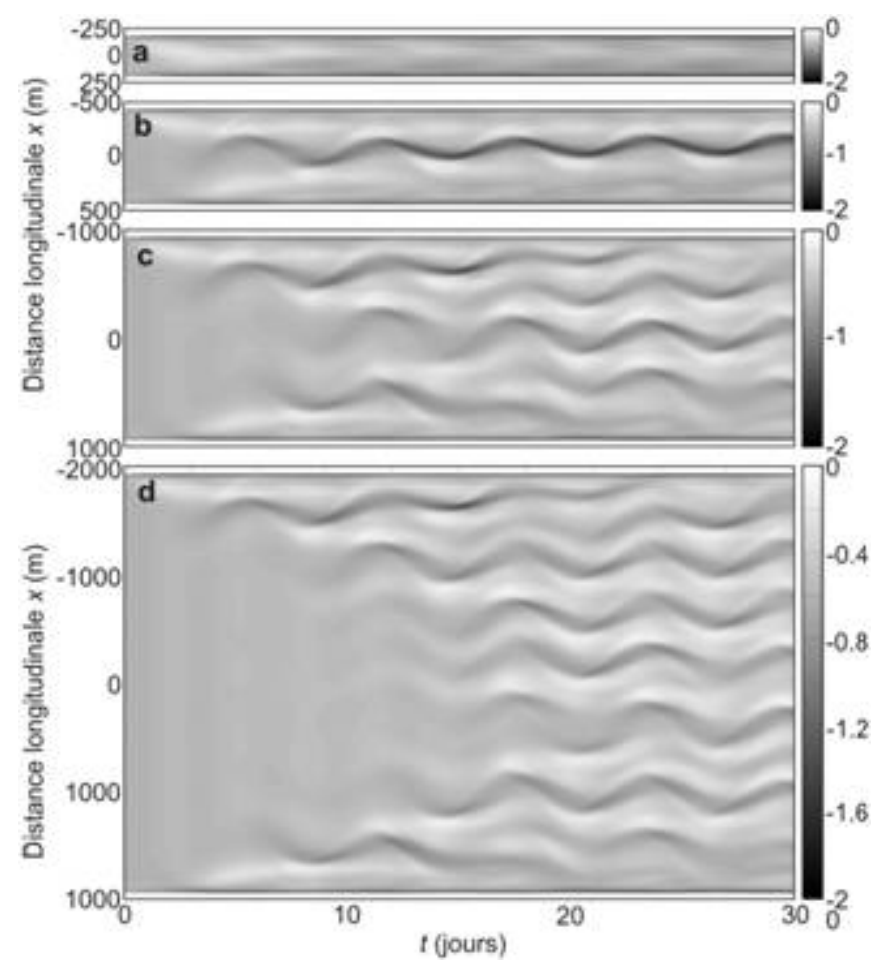

Figure 4. Evolution temporelle du profil de plage Zb pour un forçage de vague instationnaire en $\theta$ (amplitude $10^{\circ}$ ) en partant de plages en baie rectilignes. (a) $L x=500 \mathrm{~m}$, (b) $L x=1000 \mathrm{~m}$, (c) $L x=2000 \mathrm{~m}$ et (d) $L x=4000 \mathrm{~m}$.

\section{Discussion et conclusions}

Même si dans notre étude les ondes de groupe, les processus de diffraction, de réflexion ou de déferlement des vagues sur les caps n’ont pas été entièrement pris en compte, l'ensemble des circulations observées dans la littérature (SHORT \& MASSELINK, 1999) ont pu être obtenues en partant de plages et de forçages de vagues très idéalisés 


\section{XII ${ }^{\text {èmes }}$ Journées Nationales Génie Côtier - Génie Civil \\ Cherbourg, 12-14 juin 2012}

(figure 5). Nos simulations montrent que les chenaux de cap sont préférentiellement observés pour les plages à faible courbure, sans claire dépendance de l'angle d'incidence des vagues, et sont favorisés par des vagues avec un étalement directionnel significatif. En effet, une étude plus détaillée (pas discutée ici) montre que les courants d'arrachement de cap, et la formation de chenaux de cap résultante, est en grande partie contrôlée par les zones d'ombre énergétiques des vagues au voisinage de chaque cap, induisant des gradients de dissipation d'énergie des vagues par déferlement bathymétrique. Les dimensions et l'importance de ces zones d'ombre énergétiques augmentent avec l'étalement directionnel des vagues au large.

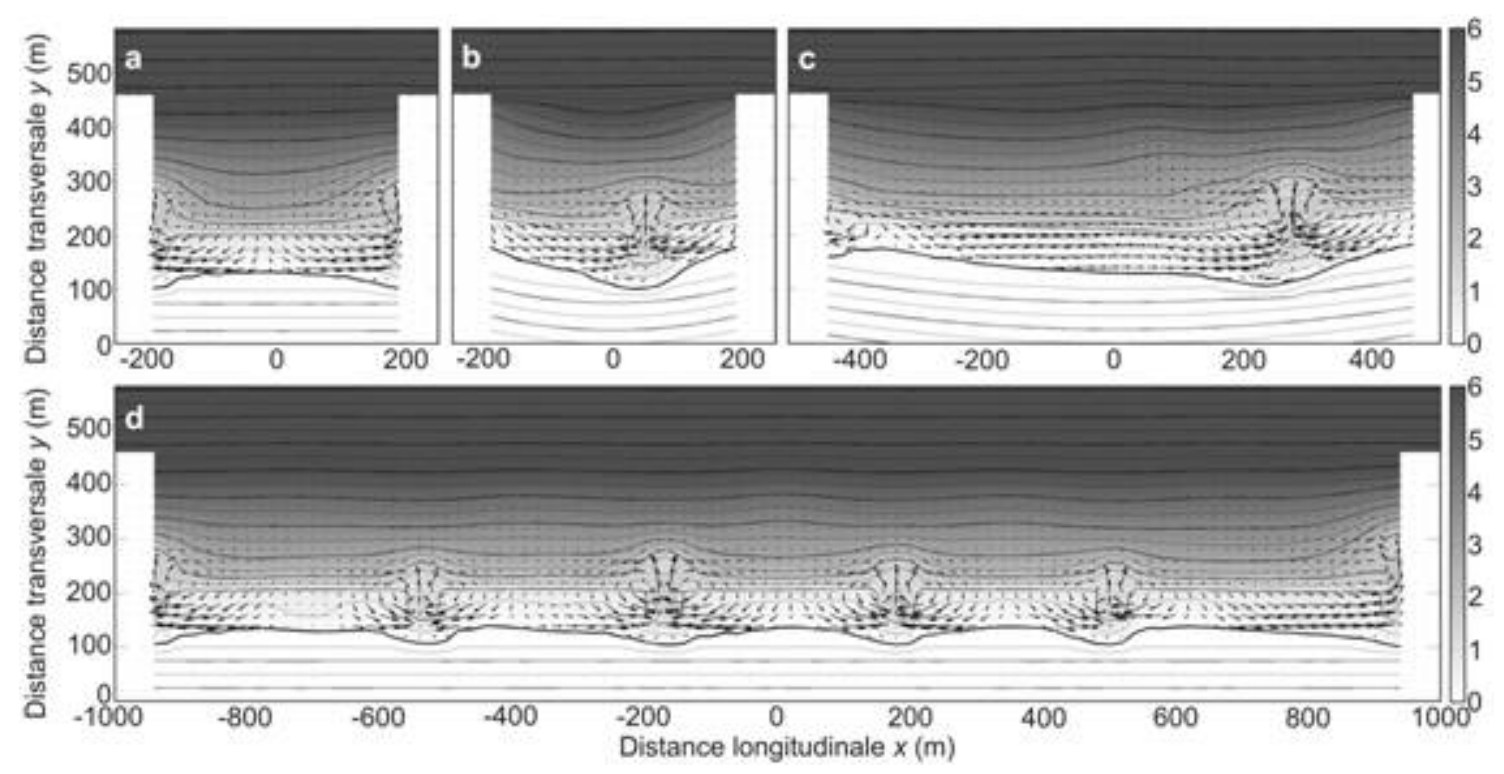

Figure 5. (a) Circulation cellulaire avec deux chenaux de cap ( $L x=500 \mathrm{~m}$, plage linéaire, $\theta=5^{\circ}$ ), (b) circulation cellulaire avec un chenal central ( $L x=500 \mathrm{~m}$, plage incurvée, $\theta=5^{\circ}$ ), (c) circulation transitoire avec un chenal de cap et un chenal central

( $L x=100 \mathrm{~m}$, plage incurvée, $\theta=5^{\circ}$ ) et (d) Circulation normale ( $L x=2000 \mathrm{~m}$, plage linéaire, $\theta=0$ )

Nos simulations suggèrent également que, pour des forçages instationnaires ou pour des forçages stationnaires avec une forte obliquité des vagues, l'influence des caps sur la morphodynamique des barres peut se propager progressivement dans l'ensemble du domaine. Une obliquité instationnaire du champ de vague induit des appariements et scissions permanentes des systèmes barre/chenal et une grande variabilité des longueurs d'onde le long de la plage. Cette variabilité le long de la plage est consistante avec l'hypothèse que les systèmes barre/chenal le long des plages en baie sont des motifs auto-organisés. Cela est en accord avec de récentes observations de la dynamique des systèmes barre/chenal. Pour une description extensive de l'étude, le lecteur est invité à consulter CASTELLE \& COCO (sous presse). 
Thème 2 - Dynamique sédimentaire

\section{Remerciements}

Ces travaux ont été réalisés et financés dans le cadre l’ANR BARBEC (ANR N²010 JCJC 602 01). GC est financé par Cantabria Campus Internacional (Augusto Gonzalez Linares Program)

\section{Références bibliographiques}

BOOIJ N., RIS R.C., HOLTHUIJSEN L.H. (1999). A third-generation wave model for coastal regions, Part 1, Model description and validation. Journal of Geophysical Research 104 (C4), pp 7649-7666. doi:10.1029/98JC02622

CASTELLE B., RUESSINK B.G. (2011). Modeling formation and subsequent nonlinear evolution of rip channels: time-varying versus time-invariant wave forcing. Journal of Geophysical Research 116 (F04008). doi:10.1029/2011JF001997

CASTELLE B., COCO G. (sous presse). The morphodynamics of rip channels on embayed beaches. Continental Shelf Research.

COCO G., MURRAY A.B. (2007). Patterns in the sand: from forcing templates to self-organization, Geomorphology, 91, pp 271-290. doi:10.1016/j.geomorph.2007.04.023

ENJALBERT C., CASTELlE B., RIHOUEY D., DAILlOUX D. (2011). Highfrequency video observation of geologically-constrained barred-beach: La Grande Plage de Biarritz (France). Journal of Coastal Research SI 64, pp 70-74.

GALLOP S.L., BRYAN K.R., COCO G., STEPHENS S.A. (2011). Storm-driven changes in rip channel patterns on an embayed beach. Geomorphology 127, pp 179-188. doi:10.1016/j.geomorph.2010.12.014

GARNIER R., CALVETE D., FALQUÉS A., DODD N. (2008). Modelling the formation and the long-term behavior of rip channel systems from the deformation of a longshore bar. Journal of Geophysical Research 113 (C07053), doi:10.1029/2007JC004632

HARLEY M.D., TURNER I.L., SHORT, A.D., RANASINGHE R. (2011). A reevaluation of coastal embayment rotation: The dominance of cross-shore versus alongshore sediment transport processes, Collaroy-Narrabeen Beach, southeast Australia. Journal of Geophysical Research 116 (F04033), doi:10.1029/2010JF001989

LOUREIRO C., FERREIRA O., COOPER J.A.G. (2012). Extreme erosion on highenergy embayed beaches: influence of megarips and storm grouping. Geomorphology 15, pp 155-171. doi:10.1016/j.geomorph.2011.10.013

RENIERS A.J.H.M., ROELVINK J.A., THORNTON E.B. (2004). Morphodynamic modeling of an embayed beach under wave group forcing. Journal of Geophysical Research 109 (C01030). doi:10.1029/2002JC001586

SHORT A.D., MASSELINK G. (1999). Embayed and structurally controlled embayed beaches. In: Short, A. D. (Ed.), Handbook of Beach and Shoreface Morphodynamics. Wiley, Chichester, pp 230-250. 